Urol. int. 1962;14:355-356

\title{
Index rerum ad Vol. 14
}

Confecit Wilhelm Baumann, Zurich

Adenoma prostatae, cystometria, 226

- $\quad$ therapia sclerotisans, 218Aspiration biopsy in prostatic cancer,

140

Bladder, damage following radiation, 65, 89

- $\quad$ isolated, 42

Boari-plastic and ureterocystoneostomy, 193

C-reactive protein in cancer of the kidneys, 250

Calcoli di urati, terapia con limoni, 28

Calculosis renal subsiguiente a las frac-turas oseas, 319

Carcinoma prostatico, metodica dia-gnostica, 140

Cavernas tuberculosas renales, 1

Citraturia et urolithiasis, 15

Chemotherapy of urogenital tuberculosis, 1

Cowper's gland, roentgenogram, 125

Cystometric study in prostatic patients, 226

Cytological examination in prostatic cancer, 140

Ectopie urétérale sur rein double, incontinence d'urine, 310

Fracturas oseas, calculosis renal subsiguiente, 319

Glandula de Cowper, la imagen radio-

grafica, 125 Glitter-cells Sternheimer-Malbin in

pyelonephritis, 160

Harnblase, Operationen an der isolier-ten, 42

- $\quad$ Spätschäden durch Strahlenbehand-lung, 65, 89

Harnleiterstumpf nach Nephrectomie,

129 Hydronephrosis, distribution of $\mathrm{Na} 24$ in

the kidney, Ill

Hypertrophia prostatae, cystometria, 226

resectio transurethralis versus therapia dilatatoria, 119

therapia sclerotisans, 218

Idronefrosi sperimentale, distribuzione

del Na24 nel rene, Ill Incontinencia de orina por ectopia

ureteral, 310 Intestinoplastiken, ihre Geschichte, 173 Ipertrofía della prostata, resezione

transuretrale e dilatazione strumen-

tale, 119

Kidney, C-reactive protein in cancer, 250

dislocation by splenic enlargement, 257

distribution of Na24 in experimental hydronephrosis, Ill

medullary spongiosis, 239 
tuberculous cavitation, 1

Lithiase rénale et fracture, 319

, solutions litholytiques, 54

Litiasis urica, metabolismo de las puri-

nas, 28 Lithiase urinaire et la cítraturie, 15 Litholytic solutions, 54

Markschwammniere, 239

Milza, dislocazioni reno-ureterali con-

seguenti a tumefazioni, 257 Milztumoren, Verlagerung von Niere

und Ureter, 257

Nefrectomia, el muñón ureteral, 129 Nephrectomy, affections of the ureteral

stump, 129 Niere, C-reaktives Protein bei Krebs,

250 -, Markschwammniere, 239 -, tuberkulöse Kaverne, 1 -, Verlagerung bei Milzschwellungen,

257 Nierenbecken, die operative Behand-

lung der Tumoren, 129

356

Index

rerum

XIV/1962

P32 in prostatic cancer, 140

Papilloma vesicae, ureterocystoneostomia, 193

Periureteritis, 292

Pielonefrite cronica, Glitzerzellen di Sternheimer-Malbin, 160

Plastic operations, the history, 173

Prostata, cystometria post prostatecto-miam, 226

-, resectio transurethralis versus thera-pia dilatatoria, 119

-, therapia sclerotísans hypertrophiae, 218

Prostatectomia, cystometria post opera-tionem, 226

Prostatic cancer, cytological examination, 140

-, diagnosis, 140

-, excretion of estrogens and 17-keto-steroids, 140

-, prostatic serum acid phosphatase, 140

-, radioactive phosphorus $\mathrm{P} 32,140$

-, trans-rectal aspiration biopsy, 140

-, vesiculography, 140

Prostatic serum acid phosphatase, 140 Pyelonephritis chronica, cellulae Sternheimer-Malbin, 160

Radiation, late damage of the bladder in

women, 65, 89 Radiographie de la glande de Cowper,

125 Radioterapia, lesiones tardias de la

vejiga femenina, 65 Rate, déplacements rénaux-urétéraux

consécutifs aux tumefactions, 257 Rein, cavernes tuberculeuses, 1 -, test à la protéine $\mathrm{C}$ reactive

dans

tumeurs malignes, 250 Rein-éponge, 239

Renal pelvis, treatment of tumors, 129 Rene, caverne tubercolari renali, 1 -, il test della proteina

C-reattiva nei

tumori maligni, 250 Rene a spugna, 239 Resectio transurethralis prostatae versus 
therapia dilatatoria, 119 Riñon, cavernas tuberculosas, 1 -, dislocaciones derivadas de tumefacciónes del bazo, 257

-, la proteina $\mathrm{C}$ reactiva en los tumores

malignos, 250 Riñon en esponja, 239

Sclerosing therapy for prostatic hypertrophy, 218

Solutiones litholyticae, 54

Splenic enlargement, reno-ureteral dislocation, 257

Sternheimer-Malbin-Glitzerzellen, 160

Strahlenbehandlung, Spätschäden an der weiblichen Harnblase, 65, 89

Tuberculose rénale, chimiothérapie, 1 Tuberculous renal caviation, 1

Urate calculosis, treatment by lemons, 28 Ureter, dislocation by splenic enlargement, 257 Ureter, Verlagerung bei Milzschwel-

lungen, 257 Ureteral stump of the nephrectomy, 129 Ureterectomia secundaria, 129

Ureterocystoneostomia, 193 Urinary calculosis vide urolithiasis Urinary incontinence in ureteral ecto-

pia, 310 Urogenital tuberculosis, chemotherapy, 1

-, renal resection, 1

-, speleotomy, 1

Urolithiasis, an indirect sequel of fracture, 319 -, purine metabolism, 28 -, solutiones

litholyticae, 54 -, treatment by lemons, 28

- $\quad$ et citraturia, 15

Vescica femminile, lesioni tardive da

raggi, 65, 89 Vescica isolata, ricostruzione della via

urinaria, 42 Vesiculography in prostatic cancer, 140 Vessie, lesions tardives après irradiation,

65,89

-. - $\quad$ isolée

reconstruction du tractus uri-naire

42

Wertheim

Ureterocystoneostomia

193 